\title{
Suggestions for the Work of Dental Hygienists and a Reflection of Health Insurance Charges according to Demographic Changes
}

\author{
Myung-Jin Lee ${ }^{1}$ and Sang-Hwan $\mathrm{Oh}^{2, \dagger}$ \\ 'Department of Dental Hygiene, Division of Health Science, Baekseok University, Cheonan 31065, \\ ${ }^{2}$ Department of Dental Hygiene, College of Medical Science, Konyang University, Daejeon 35365, Korea
}

\begin{abstract}
Given that the demand for dental care for elderly individuals is expected to increase according to demographic changes, long-term roadmaps for the current health insurance system should be actively planned. The study aimed to investigate the health insurance system in Korea, compared to that of Japan, and suggest directions for the work of dental hygienists to provide basic data on efficient improvements in the health insurance system. Based on the collected data, the website was used to collect additional related data from Korea and Japan. The most common cause of death in Korea was malignant neoplasms, and most patients were hospitalized for diseases and disorders of the musculoskeletal system and connective tissue. Dental care covered by Korean medical insurance for individuals aged 65 years or older was treatment oriented, such as dentures and implants, while in Japan, treatment-intensive items, such as visiting a dental hygienist for guidance and provision of home-care professional oral hygiene treatment, were covered. The percentages of remaining teeth in Korea and the percentage of remaining 20 teeth were $68.9 \%$ in those aged 65 to 74 years and $51.2 \%$ in those aged 75 to 84 years in Japan. A strategy for promoting oral health among elderly individuals should be established, and a multilateral intervention approach is required to prevent oral problems from leading to deterioration of whole-body health. The role of dental hygienists in providing comprehensive assessment to elderly individuals is important. In order to provide systemic oral care, it is necessary to introduce oral care systems according to the national policy.
\end{abstract}

Key Words: Aged, Demographic aging, Dental care, Insurance, Oral health

\section{Introduction}

The characteristics of the Korean population by era show that the population surged around 1960 due to the high birth rate. Between the 1960s and 1990s, the rate of population growth slowed as the birth rate decreased because of family planning, improvement in education, and the entry of women into the society ${ }^{1,2)}$. Since the 2000 s, the birth rate has declined due to changes in the value of marriage and avoidance of childbirth; furthermore, an increase in life expectancy owing to developments in medicine and the economic growth led to aging ${ }^{3)}$.
According to the National Health Insurance Service's "National Health Insurance Statistical Yearbook," the medical expense for elderly individuals' in 2017 was 28.3 trillion won, which was approximately twice that of the expenditure much in 2010. Considering that the average annual medical expenses per senior citizen is 4.2 million won, an increase in the elderly population leads to an increase in their medical expenses ${ }^{4)}$. According to data from the Organization for Economic Cooperation and Development (OECD) Health in 2019, out-of-pocket ratios of the medical expenses in Korea and Japan were $32.9 \%$ and $12.7 \%$, respectively, where the ratio for Korea 
was more than twice that for Japan ${ }^{5}$.

One of the problems associated with a rapidly aging population is that the number of elderly individuals with chronic diseases also increases ${ }^{6}$. In fact, the prevalence of chronic diseases in elderly individuals aged 65 years and older is reported to be approximately $90 \%{ }^{7}$. Since chronic diseases are an urgent problem that require continuous and systematic management to prevent complications, a welfare policy for elderly individuals to manage diseases over a long period is necessary ${ }^{3}$.

In Korea, the Medical Insurance Act was enacted on December 16, 1963, and the National Health Insurance Act was enacted on February 8, 1999, expanding on the prevention and rehabilitation of diseases and injuries. On July 2, 2008, long-term care insurance for elderly individuals was implemented, aiming to stabilize their aging lives ${ }^{6}$. However, the scope and level of benefits, some of the criteria for judging the quality and contents of the system, are still treatment-oriented ${ }^{8)}$. In particular, the dentistry is weaker than that in other areas. Since most dental prostheses are classified as non-beneficial, it has been reported that a large proportion of the population are left with dental defects due to large medical expenses ${ }^{1,8)}$. As such, the current medical insurance system is not suitable for the provision of examinations, disease prevention, and promotion of health; thus, improvements in these areas are necessary. With the rapid change in the age group, strategies for mid- to long-term dental services must be prepared.

Since the introduction of long-term care insurance for elderly individuals, Japan has implemented various measures against aging. A new field of dental-oral life care for elderly individuals has been introduced, and its maintenance and systemization is ongoing ${ }^{9}$. For the long-term view of promoting oral health in individuals, a rational medical insurance system is necessary, and after scientific data has shown that oral health is closely related to general health, awareness of the necessity of the dental care system is rapidly increasing ${ }^{10)}$.

Currently, Korea needs the knowledge and experience that has accumulated in countries with aging-associated problems. We investigated dental care in Japan, which has been experiencing aging problems before Korea and suggest the direction of the work of dental hygienists by providing basic data regarding how to effectively improve the dental care system.

\section{Methods}

For this study, data from the following sources were collected: 2015 National Medical Care Resources and Utilization Survey from the Ministry of Health and Welfare; cause-of-death statistics from 2007 to 2017 and number of hospitalized patients by diagnosis-related

Table 1. Trends of Causes of Death, 2007 2017 (Unit: 1 Per 100,000 People, \%)

\begin{tabular}{|c|c|c|c|c|c|c|}
\hline & \multicolumn{2}{|l|}{2007} & \multicolumn{2}{|l|}{2016} & \multicolumn{2}{|l|}{2017} \\
\hline & Cause of death & Rate of death & Cause of death & Rate of death & Cause of death & Rate of death \\
\hline 1 & Malignant neoplasm & 138.1 & Malignant neoplasm & 153.0 & Malignant neoplasm & 153.9 \\
\hline 2 & $\begin{array}{l}\text { Cerebrovascular } \\
\text { disease }\end{array}$ & 60.0 & Heart disease & 58.2 & Heart disease & 60.2 \\
\hline 3 & Heart disease & 44.1 & $\begin{array}{l}\text { Cerebrovascular } \\
\text { disease }\end{array}$ & 45.8 & $\begin{array}{l}\text { Cerebrovascular } \\
\text { disease }\end{array}$ & 44.4 \\
\hline 4 & Intentional self-harm & 24.9 & Pneumonia & 32.2 & Pneumonia & 37.8 \\
\hline 5 & Diabetes melllitus & 23.1 & Intentional self-harm & 25.6 & Intentional self-harm & 24.3 \\
\hline 6 & Transport accident & 15.6 & Diabetes melllitus & 19.2 & Diabetes melllitus & 17.9 \\
\hline 7 & $\begin{array}{l}\text { Chronic lower } \\
\text { respiratory disease }\end{array}$ & 15.4 & $\begin{array}{l}\text { Chronic lower } \\
\text { respiratory disease }\end{array}$ & 13.7 & Liver disease & 13.3 \\
\hline 8 & Liver disease & 15.0 & Liver disease & 13.3 & $\begin{array}{l}\text { Chronic lower } \\
\text { respiratory disease }\end{array}$ & 13.2 \\
\hline 9 & Hypertensive disease & 11.1 & Hypertensive disease & 10.6 & Hypertensive disease & 11.3 \\
\hline 10 & Pneumonia & 9.4 & Transport accident & 10.1 & Transport accident & 9.8 \\
\hline
\end{tabular}


groups in 2015 from Statistics Korea; oral health status of elderly individuals aged 65 years and older from the Korean Centers for Disease Control \& Prevention; Medical Insurance Report from the Korea National Health Insurance Service; and Japan's Ministry of Health, Labor, and Welfare website.

\section{Results}

\section{Causes of death and chronic diseases among hospitalized patients in Korea}

The top 10 causes of death were malignant neoplasms (cancer), heart disease, cerebrovascular diseases, pneumonia, intentional self-harm (suicide), diabetes, liver diseases, chronic lower respiratory disease, hypertensive diseases, and transport accidents (Table 1$)^{11)}$. The highest percentage of malignant neoplasms occurred in the bronchus and lungs. The causes of death that increased compared to 10 years prior were heart disease, pneumonia, and liver diseases; those that decreased were cerebrovascular disease, intentional self-injury, diabetes, chronic lower respiratory disease, and transport accidents. The number of cases of pneumonia continued to increase from the 10th highest in 2007 to the 4th highest since 2016 compared to the number of cases of other causes. The highest numbers of hospitalized patients by disease group were due to

Table 2. Number of Hospitalized Patients by Disease Group

\begin{tabular}{lc}
\hline \multicolumn{1}{c}{ Disease } & $\begin{array}{c}\text { Number of } \\
\text { hospitalized patients }\end{array}$ \\
\hline $\begin{array}{l}\text { Disease and disorder of the } \\
\text { musculoskeletal system and } \\
\text { connective tissue }\end{array}$ & $2,099,286$ \\
Disease and disorder of digestive system & $1,336,384$ \\
Disease and disorder of respiratory & 979,819 \\
system & \\
Disease and disorder of ear, nose, & 607,271 \\
mouth and throat & 568,392 \\
Disease and disorder of nervous system & 517,692 \\
Disease and disorder of eye & 393,706 \\
Disease and disorder of & \\
circulatory system & 379,828 \\
Disease and disorder of the & \\
hepatobiliary system and pancreas & 9,340 \\
Pre-mdc &
\end{tabular}

mdc: major diagnostic category. conditions of the musculoskeletal and connective tissue; digestive system; respiratory system; ear, nose, mouth, and throat systems; nervous system; eyes; circulatory system; hepatobiliary system and pancreas; and pre-major diagnostic category (Table 2$)^{12)}$.

\section{Types of dentistry covered by dental care for individuals aged 65 years and older}

Comparison of health insurance of 65 years and over between Korea and Japan is shown in Table 3.

\section{1) Korea}

\section{(1) Dentures}

Dentures are covered by health insurance benefit costs part 1 , costs relative value scores part 2, and the relative score and calculation guidelines in chapter 18: "complete resin dentures, temporary complete resin dentures, partial dentures, and temporary partial dentures (based on three values)." Indications for complete dentures (resin or metal) in the upper jaw or lower jaw are fully edentulous patients, and those for partial metal dentures are patients who can receive partial dentures using the remaining teeth due to tooth defects in the upper or lower jaw (partial or multiple). According to the Health Insurance Review and Assessment Service, the coverage applies once every 6 years and the co-pay for individuals aged older than 65 years is $30 \%{ }^{13)}$.

\section{(2) Implants}

Implants are covered by health insurance benefit costs part 1 , costs relative value scores part 2 , and the relative score and calculation guidelines in chapter 18: "Chapter

Table 3. Comparison of Health Insurance of 65 Years and Over between Korea and Japan

\begin{tabular}{ccc}
\hline Insurance & Korea & \multicolumn{1}{c}{ Japan } \\
\hline Health insurance & Denture & $\begin{array}{c}\text { Medical assistance of } \\
\text { dental visit } \\
\text { Dental hygiene guidance } \\
\text { of visit }\end{array}$ \\
& Implant & $\begin{array}{l}\text { Oral hygiene treatment } \\
\text { specializing in }\end{array}$ \\
& & rehabilitation patients \\
& & such as home care \\
Long-term care insurance & & Home care management \\
& & guidance \\
\hline
\end{tabular}


11. Implant section." Partially edentulous patients who require dental implants with non-precious metal ceramic prosthetic restorations using separate implants (fixed bodies, abutments) in the jawbone are covered. According to the Health Insurance Review and Assessment Service, the coverage applies twice per person, and the co-pay for individuals aged older than 65 years is $30 \%{ }^{13)}$.

\section{2) Japan}

\section{(1) Health insurance}

Dental visits and education from dental hygienists are intended for patients who are unable to visit the hospital and live within a $16-\mathrm{km}$ radius ${ }^{14)}$.

\section{A. Dental visits}

Dental visits can cost between 300 and 1,150 yen depending on the number of individuals and time. The costs add up if a dental hygienist carries out medical treatments with a dentist, and in these cases, the dental hygienist's name must be included on the medical record card.

B. Educational visits from dental hygienists

According to the dentist that performs a dental consultation, a dental hygienist, public health nurse, nurse, or para-nurse may visit and provide on-site education on oral care and denture cleaning, as well as on restoring and maintaining patients' oral function to patients in a single building or to families for at least 20 minutes. Depending on the number of patients in a single building, the cost can total up to 3,000 to 3,600 yen. The visits can be scheduled four times a month per patient.

C. Professional oral hygiene treatment for the care of patients at home

The costs add up if a dental hygienist is accompanied by a doctor to perform professional oral hygiene treatment. The cost is 1,200 yen per patient per month when professional oral cleaning, denture cleaning, or mechanical denture cleaning of the teeth, tongue, and oral mucosa are performed using oral cleaning equipment.

\section{(2) Long-term care insurance}

Long-term care insurance is intended for individuals aged 65 years and older, with some aged 40 years and older. The co-pay is $10 \%$, and the conditions are use of a nursing facility, care consultation, and visits.

A. Home care management

The costs add up when patients in a single building or a family is provided with on-site instructions for oral denture cleaning and oral functions for 20 minutes or longer. Depending on the number of patients in a single building, the costs can total up to 3,000 to 3,600 yen. The visits can be scheduled within 3 months after a dentist's appointment and 4 times a month per patient.

\section{Number of remaining teeth in patients aged older than 65 years}

\section{1) Korea}

According to the National Health and Nutrition Survey data (2007 2013), the percentages of elderly individuals aged older than 65 years who possessed more than 20 natural teeth were $46.65 \%$ in 2007 and $48.00 \%$ in 2013 . The average numbers of retained teeth were 15.8 in 2007 and 16.4 in 2013 (Table 4) ${ }^{15)}$.

\section{2) Japan}

According to the survey on dental diseases by Japan's Ministry of Health, Labor, and Welfare, the proportion of elderly individuals with more than 20 remaining teeth generally increased for all ages, and in particular, more than $50 \%$ of elderly individuals aged 75 to 84 years had more than 20 teeth (Table 5).

Table 5. Percentage of Over 20 Remaining Natural Teeth by Age Group in Japan

\begin{tabular}{cccccc}
\hline Age $(\mathrm{y})$ & 1993 & 1999 & 2005 & 2011 & 2016 \\
\hline $65 \sim 74(\%)$ & 28.9 & 41.5 & 50.1 & 60.4 & 68.9 \\
$75 \sim 84(\%)$ & 10.6 & 16.3 & 25.0 & 40.2 & 51.2 \\
\hline
\end{tabular}

Table 4. Percentage of 20 or More Natural Teeth and Average of Remaining Natural Teeth in Adults Aged 65 and Over (2007 2013)

\begin{tabular}{|c|c|c|c|c|c|c|c|}
\hline Teeth & 2007 & 2008 & 2009 & 2010 & 2011 & 2012 & 2013 \\
\hline Percentage of 20 or more natural teeth $(\%)$ & 46.65 & 44.65 & 45.00 & 46.95 & 46.55 & 47.70 & 48.00 \\
\hline Average of remaining natural teeth (n) & 15.8 & 15.9 & 15.9 & 16.0 & 16.3 & 16.5 & 16.4 \\
\hline
\end{tabular}




\section{Discussion}

Due to the rapid aging in Korea, the proportion of elderly individuals in the population is expected to rapidly increase from $20 \%$ in 2020 to $41 \%$ in 2060 , increasing the need for a welfare policy for elderly individuals ${ }^{5)}$. As shown in Table 1, the leading cause of death according to the cause-of-death statistics from the Korean Statistical Information Service (KOSIS) is malignant neoplasms (cancer), among which lung cancer had the highest mortality rate. It is also worth noting that the number of cases of pneumonia has gradually risen from the 10th highest in 2005 to the 4th highest in 2017, compared to the number of cases due to other causes ${ }^{11)}$. The OECD has predicted an increase in the life expectancy when the major causes of death are eliminated, and the KOSIS data suggest that life expectancy can be increased by one year if pneumonia is eliminated as a cause of death ${ }^{5,11)}$. In addition, several studies have shown that the incidence of aspiration pneumonia has reduced because of proper oral care, emphasizing the role of oral hygiene management in disease prevention ${ }^{16)}$. The number of hospitalized patients by disease group, as shown in Table 2, was the highest for conditions of the musculoskeletal system, followed by the digestive and respiratory systems ${ }^{12)}$. Poor nutritional intake due to poor oral function among elderly individuals leads to a decrease in muscle mass and muscle strength, leading to problems in the musculoskeletal system and physical performance ${ }^{17)}$. Therefore, the association between musculoskeletal disorders and oral diseases, which are the main causes of hospitalization, is considered a problem that cannot be overlooked.

As individuals age, they become more vulnerable to various diseases, and declining oral health leads to a decrease in chewing ability, which may affect mental health such as that due to stress from nutritional imbalances, increased risk of developing multiple diseases due to digestive disorders, dietary restrictions, and a loss of self-confidence ${ }^{18)}$. Thus, oral health is an important factor in determining the quality of life in old age ${ }^{19-21)}$. In 1989, when Japan began to notice the problems associated with aging, they initiated the 8020 movement with the aim of having 20 teeth at the age of 80 years, led by the Dental
Society and Ministry of Health, Labor, and Welfare. In 2000, the 8020 foundation was launched to activate private projects, and dental visits were initiated to implement the 8020 movement. According to the survey on dental disease that the Ministry of Health, Labor, and Welfare conducts every 5 to 6 years, the achievement rate of the movement increased from $10.6 \%$ in 1993 to $25.0 \%$ in $2005,40.2 \%$ in 2011 , and $51.2 \%$ in 2016 (Table 5) ${ }^{14)}$. In 2016, the percentage increased by more than five times since 1993, where more than half of all elderly individuals aged 80 years had more than 20 teeth. According to the National Health and Nutrition Survey of the Korean Centers for Disease Control and Prevention, half of all elderly individuals in Korea had less than 20 teeth in 2013. Even in 2015, the number of teeth remaining in elderly individuals aged above 65 years was 16 to $17^{22)}$. Dental health can also be used as an indicator of systemic health. A previous study of 1,843 seniors aged 65 years and above found that the number of remaining teeth affected bone health. By analyzing the correlation between the number of remaining teeth in elderly individuals and their bone health, elderly individuals with less than 20 remaining teeth who receive prosthetic treatment have a 1.5 times higher risk of osteopenia and a 2.6 times higher risk of osteoporosis compared to that in elderly individuals with more than 20 remaining teeth ${ }^{23)}$. This may be because elderly individuals who experience chewing difficulties only select soft foods that are easy to chew, leading to a deficiency of nutrients such as calcium ${ }^{10)}$. Without teeth, elderly individuals not only receive unhealthy nutrition, but also become prone to systemic diseases. However, discontinuation of dental treatment considering the loss of teeth as a part of aging leads to delay in treatment for economic reasons, which can affect the health of the whole body ${ }^{1}$. In addition, previous studies have demonstrated a strong association between the loss of teeth and systemic risk factors, such as malnutrition, obesity, diabetes, chronic obstructive pulmonary disease, pneumonia, cognitive impairment, and mortality risk $^{24)}$. As oral disease is a socially important problem that not only reduces an individual's health and quality of life, but also requires high medical expenses, it is important to pay close attention to the management of symptoms and the 
medical history of oral diseases rather than the treatment ${ }^{25)}$.

According to a 2014 survey of elderly individuals, more than half of all elderly individuals who experienced discomfort in sensory function felt more discomfort while chewing over their eyesight and hearing ${ }^{26)}$. Since the proportion of elderly individuals aged 65 years or older who experienced discomfort caused by the deterioration in physical function felt the most discomfort due to their chewing ability, more active counter measures to improve chewing ability among elderly individuals are necessary. On evaluation of the chewing ability in previous studies, the number of remaining teeth and various oral functional factors were found to be complexly related ${ }^{27)}$. Rather than applying only one method in elderly individuals experiencing physical changes due to aging, care with more comprehensive improvement and satisfaction should be provided $^{28)}$. In Japan, the concept of "deterioration in oral function" was created to not only reflect the dental condition, but also the oral filth, dryness, occlusion, deterioration of the tongue and lips, reduced tongue pressure, and reduced chewing and swallowing abilities. In 2018, medical insurance coverage for the management of oral dysfunction became available, and oral functional management is offered following oral functional tests. Dental hygienists can perform tests and manage oral function under the guidance of a dentist. However, in Korea, many medical treatments do not have come under insurance coverage, and their accessibility is failing due to high medical expenses. In addition, the health care system consists of dentures and implants, which focuses more on the treatment rather than prevention of oral diseases ${ }^{29)}$. However, there are many preventive care items in Japan, and based on the methodology of dental care for elderly individuals, methods of oral hygiene as well as dental supplies and medicines to be used are established ${ }^{9)}$. In addition, dental hygienists provide professional oral hygiene measures and dental health education for patients in nursing homes. This may be related to the increase in the number of remaining teeth among elderly individuals in Japan.

Although the costs of visiting dental hygienists are relatively high compared to the medical expenses of dentists, the Ministry of Health, Labor, and Welfare has initiated these visits. This is expected to help not only improve oral health, but also relieve employment shortages and provide re-employment for individuals who are in between jobs. The impact of demographic changes leads to changes in disease patterns and proportions; thus, the health insurance structure will need to change accordingly. Therefore, in Japan, efforts have been implemented to enable the early detection of diseases and prevention of chronic diseases through preventive care. As the Korean population is rapidly aging, it is necessary to implement efforts to reduce the costs of treatments with a paradigm shift from treatment to prevention (Table 3 ).

Based on these results, if dentists and dental hygienists can diagnose and prevent oral diseases using devices that can verify the diagnosis or symptoms of patients, this can contribute to improvements in the oral health and quality of life among elderly individuals. The role of dentists and dental hygienists in providing comprehensive assessment and access to elderly individuals is important, and it is necessary to introduce oral care systems in accordance with national policies to provide continuous and systematic oral care ${ }^{30)}$. In particular, the long-term care roadmap for the current dental care system must be actively planned, given that the demand for dental care among elderly individuals is expected to increase as life expectancy increases ${ }^{31)}$. A multilateral interventional approach is necessary to prevent oral problems leading to a deterioration in overall health, and studies on the establishment of a healthcare system is necessary for the number of acts that guarantee the activities of dentists and dental hygienists, as well as changes in the paradigm shift from treatment to prevention.

\section{Notes}

\section{Conflict of interest}

No potential conflict of interest relevant to this article was reported.

\section{Ethical approval}

This study did not require IRB review because it was a review article. 


\section{Author contributuins}

Conceptualization: Sang-Hwan Oh, Myung-Jin Lee. Data acquisition: Myung-Jin Lee. Supervision: Sang-Hwan Oh. Writing-original draft: Myung-Jin Lee. Wrting-review \& editing: Sang-Hwan Oh.

\section{ORCID}

Myung-Jin Lee, https://orcid.org/0000-0002-9267-4947

Sang-Hwan Oh, https://orcid.org/0000-0002-5944-0129

\section{References}

1. Kim JH, Park YD: Dental service provider's recognition of national health insurance coverage of denture. J Korean Acad Oral Health 33: 644-652, 2009.

2. Lee MJ, Kim DK, Hwang SJ, Oh SH: Elderly welfare system and role of dental hygienists in Korea and Japan. J Dent Hyg Sci 18: 172-181, 2018. https://doi.org/10.17135/jdhs.2018.18.3.172

3. Cheong CL, Lee TJ: Population ageing and increasing health care expenditures - counting on death related costs by age group. Korean J Health Econ Policy 23: 127-148, 2017.

4. Moon JY, Park EC: Trend of benefit expenditure in national health insurance by age group, 2001-2016. Health Policy Mange 27: 372-374, 2017.

5. Organisation for Economic Co-operation and Development: OECD health statistics 2019. Retrieved August 30, 2019, from http://www.oecd.org/els/health-systems/health-data.htm (2019, July 2).

6. Koh JJ: A review of long-term care insurance for the elderly by access to an aging society. Law Policy Rev 24: 27-61, 2018.

7. Korean Statistical Information Service: The survey on the actual conditions of older person in Korea. Retrieved September 10, 2019, from http://kosis.kr/statHtml/statHtml. do?orgId=117\&tblId=DT_117071_018\&conn_path=I2(2019, February 28).

8. Hwang JM, Kim JH, Park YD: Dental service providers' satisfaction with dental health insurance. J Korean Acad Oral Health 34: 214-221, 2010.

9. Shin RH, Bae EK, Choi SH, Park II, Ohyama T, Chung MK: Analysis of current nursing homes in Korea and to assess the role and prospect of dentists in comparison to Japanese long term care insurance system. J Korean Acad Prosthodont 46: 83-91, 2008.

10. Walls AW, Steele JG: The relationship between oral health and nutrition in older people. Mech Ageing Dev 125: 853-857, 2004. https://doi.org/10.1016/j.mad.2004.07.011

11. Korean Statistical Information Service: Deaths by city \& province/cause(103 item)/by sex. Statistics Korea, causes of death statistics. Retrieved December 22, 2019, from http:// kosis.kr/statHtml/statHtml.do?orgId=101\&tblId=DT_1B34E 12\&conn_path=I2\&language $=e n(2019$, September 24).

12. Korean Statistical Information Service: Number of patients by residence and disease group. Retrieved September 10, 2019, from http://kosis.kr/statHtml/statHtml.do?orgId=117\& tblId=DT_117049_A057\&conn_path=I2(2019, February 28).

13. Health Insurance Review and Assessment Service: Health insurance review and assessment service. Retrieved February 28, 2019, from https://www.hira.or.kr/.

14. Ministry of Health, Labour and Welfare (JP): Ministry of Health, Labour and Welfare. Retrieved February 28, 2019, from https://www.mhlw.go.jp.

15. Lee HL, Lee HM, Kim HJ, Oh KW: Trends in oral health status among adults over 65 years old in Korea, 2007-2013. Public Health Wkly Rep 8: 735-736, 2015.

16. So JS: A study on the amendments of long-term care-related legislations for the introduction of part-time facility dentists. $\mathbf{J}$ Korean Dent Assoc 53: 696-704, 2015.

17. Scannapieco FA, Shay K: Oral health disparities in older adults: oral bacteria, inflammation, and aspiration pneumonia. Dent Clin North Am 58: 771-782, 2014. https://doi.org/10.1016/j.cden.2014.06.005

18. Murakami M, Hirano H, Watanabe Y, Sakai K, Kim H, Katakura A: Relationship between chewing ability and sarcopenia in Japanese community-dwelling older adults. Geriatr Gerontol Int 15: 1007-1012, 2015. https://doi.org/10.1111/ggi.12399

19. Laudisio A, Milaneschi Y, Bandinelli S, Gemma A, Ferrucci L, Incalzi RA: Chewing problems are associated with depression in the elderly: results from the InCHIANTI study. Int J Geriatr Psychiatry 29: 236-244, 2014. https://doi.org/10.1002/gps.3995

20. Cho MJ: The relationship between masticatory ability and the quality of life oral health- related using OHIP-14 of the 
elderly. J Digit Converg 14: 341-348, 2016.

https://doi.org/10.14400/JDC.2016.14.9.341

21. Lee HO, Park JY: Relationship between oral health behavior and happiness index in elderly people. J Dent Hyg Sci 16: 415-423, 2016. https://doi.org/10.17135/jdhs.2016.16.6.415

22. Kim JS, Kim SY, Jun EJ, Jeong SH, Kim JB: The number of existing permanent teeth and the denture status of elderly adults aged 65 years and above living in metropolitan cities using data from the Korean National Health and Nutrition Examination Survey. J Korean Soc Dent Hyg 18: 921-932, 2018.

23. Cho YY: Relationship between the number of remaining teeth and bone health status among the elderly in Korea. J Korean Soc Dent Hyg 18: 205-215, 2018.

24. Kim JS: The study on the relations of oral health and general and mental health over 65's adults contracting in cardiovascular disease. J Korean Soc Oral Health Sci 5: 1-8, 2017.

25. Lee KY, Lim SR: Effect of professional oral healthcare program on the oral status of elderly residents in long-term care facilities. J Dent Hyg Sci 16: 432-441, 2016. https://doi.org/10.17135/jdhs.2016.16.6.432

26. Moon SH, Hong GRS: Predictors of chewing discomfort among community-dwelling elderly. J Korean Acad Community Health Nurs 28: 302-312, 2017.

27. Ohara Y, Hirano H, Watanabe Y, et al.: Factors associated with self-rated oral health among community-dwelling older Japanese: a cross-sectional study. Geriatr Gerontol Int 15: 755-761, 2015.

https://doi.org/10.1111/ggi.12345

28. Jung HJ, Min YG, Kim HJ, et al.: Factors affecting objective and subjective masticatory ability assessment of Korean elderly people. J Korean Acad Oral Health 42: 216-223, 2018.

29. Kim MJ, Lee HK: Relationship of dental health assessment to the number of existing permanent tooth in senior citizens visited a dental hospital or clinic from some regions. J Dent Hyg Sci 7: 161-166, 2007.

30. Koong HS, Seo KW: The analysis of research trends of elderly in the dental hygiene discipline. J Dent Hyg Sci 18: 201-209, 2018.

https://doi.org/10.17135/jdhs.2018.18.4.201

31. Paik HR, Kim SY, Jin BH, Lee JY, Lim YW, Kim YJ: Developing oral health service delivery system for the disabled. J Korean Assoc Disabil Oral Health 14: 111-120, 2018. https://doi.org/10.12655/KADH.2018.14.2.111 